\title{
A ANÁLISE DO COMPORTAMENTO E AS PROPOSIÇÕES DA CONSTRUÇÃO SOCIAL DO CONHECIMENTO
}

\section{BEHAVIOR ANALYSIS AND THE PROPOSITIONS OF THE SOCIAL CONSTRUCTION OF KNOWLEDGE}

\author{
Elizeu Batista BORLOTI* \\ Zeidi Araujo TRINDADE**
}

\begin{abstract}
RESUMO
Atualmente há um grande interesse dos psicólogos em analisar a construção social do conhecimento, sempre a partir de uma perspectiva psicossocial. Os psicólogos comportamentais, influenciados pelo pensamento skinneriano, têm dado a sua contribuição. Este trabalho apresenta algumas das proposições da Análise do Comportamento acerca do conhecimento socialmente construído, discutindo as direções apontadas na análise do "conhecer socialmente" e as implicações desta análise para uma integração entre analistas de comportamento e psicólogos sociais.
\end{abstract}

Palavras-chave: Análise do Comportamento, Conhecimento Social, Comportamento Social, Práticas Culturais.

\begin{abstract}
Nowadays there is a great interest of psychologists in analyzing the social construction of knowledge, always in a psychosocial perspective. Behaviorist psychologists, influenced by the skinnerian thought, have given their contribution. This work introduces some propositions of the Behavior Analysis concerning socially built knowledge and discusses the directions pointed out in I the analysis of "to know socially" and the implications of this analysis for an integration between behavior analysts and social psychologists.

Word-key: Behavior Analysis, Social Knowledge, Social Behavior, Cultural Practices.
\end{abstract}

\footnotetext{
(*) Departamento de Psicologia Social e do Desenvolvimento, UFES; Programa de Pós-Graduação em Psicologia Social da PUC/SP. Correspondência: Av. MestreÁlvaro, 448 - Manguinhos, Serra/ES -CEP 29.168-200 - E-mail: zeidi@ globo.com (*) Departamento de Psicologia Social e do Desenvolvimento, UFES; Programa de Pós-Graduação em Psicologia, UFES. Endereço para correspondência com o Editor: Tele-fax: (027)335.2505 - e-mail: zeidi@ globo.com Informação pertinente: Trabalho derivado da dissertação de mestrado de Elizeu Batista Borloti, orientado pela Dra. Zeidi Araujo Trindade, do Programa de Pós-Graduação em Psicologia, da Universidade Federal do Espírito Santo.
} 


\section{TEXTO NA ÍNTEGRA}

Em pleno auge da chamada Revolução Cognitiva, as considerações de OVERSKEID (1995) sobre o debate entre cognitivistas e behavioristas radicais apontam, novamente, para uma questão histórica da psicologia: a organização desta ao redor do comportamento humano (ou de aspectos dele) que alguns chamam de mente, (in)consciente, idéia, cultura, crença, raciocínio, esquemas, representações, etc. Uma dessas muitas nomeações do comportamento que interessa diretamente aos propósitos desse artigo é o chamado "conhecimento construído socialmente" que tem atraído os psicólogos sociais e cuja natureza suposta justificaria, por exemplo, a necessidade de abordagens cognitivistas e/ou psicossociais. O objetivo deste trabalho é resgatar alguns elementos para uma análise comportamental deste tipo de conhecimento.

Certamente, natureza,causas e definição do comportamento têm levado muitos teóricos a julgarem que os analistas do comportamento estariam negando alguns - ou muitos - dos fenômenos humanos, geralmente condensados nas palavras "psíquicos", "cognitivos", "subjetivos" ou "psicossociais". Quanto a este último aspecto, talvez o próprio SKINNER - um dos mais influentes psicólogos deste século - tenha acirrado esta percepção sugerindo, inicialmente e aparentemente, um possível individualismo na proposição behaviorista radical ao afirmar que, num grupo, "é sempre o indivíduo que se comporta, e se comporta com o mesmo corpo e de acordo com os mesmos processos usados em uma situação não social" (SKINNER,1978a: 285-286).

Entretanto, SKINNER escreveu isto há 45 anos atrás. Desde então, a análise comportamental avançou em pesquisa e teoria. Mas é ainda na obra Ciência e Comportamento Humano (a mesma que contém o motivador das críticas) que o próprio SKINNER retifica $O$ argumento sobre um suposto isomorfismo entre o fenômeno individual e o social ao afirmar que os princípios e as categorias de análise usadas pelo analista comportamental podem contribuir para a análise dos cientistas [psicólogos] sociais sem que estes tenham que voltar-se para o comportamento individual, "pois, um outro nível de descrição pode também ser válido, e bem pode ser mais conveniente" (SKINNER,1978a:286). Este nível de descrição é perseguido pelos psicólogos sociais.

Nas pesquisas mais atuais em Psicologia Social é enfatizado que os conhecimentos socialmente construídos se integram mutuamente, englobando o comportamento dos indivíduos e os fatos sociais em sua história (SÁ, 1995). Atualmente, essa interação pode ser entendida a partir da noção de sujeito operante da filosofia skinneriana (MICHELETTO e SÉRIO, 1993). Entretanto, os efeitos da proposição da forma como se dá o "modo de conhecer" nas interações sociais, e o descontentamento de MOSCOVICl (1978) com a "psicologia S-R", permanecem ecoando até hoje na idéia da "construção" do conhecimento, opondo-se veementemente à idéia de "resposta a estímulos", presente na "representação" de algumas versões do Behaviorismo.

Um rastreamento teórico apontaria que a dimensão social do comportamento-conhecimento, na visão skinneriana, pode ser atestada, principalmente, nas proposições acerca das relações entre o comportamento encoberto (o sentir, o pensar, etc.) e as práticas sócios-culturais (TOURINHO, 1997a).Há neste ponto, evidentemente, uma posição contrária às "psicologias do indivíduo" que remetem à dicotomia sujeito-social.

Em certo sentido, para os analistas de comportamento, qualquer "psicologia do indivíduo" não poderá explicar todo e qualquer comportamento humano, assim como uma "psicologia social" não poderá explicar todo $e$ qualquer comportamento humano. Entretanto, se se focaliza a análise sobre um repertório particular, a argumentação muda. A subjetividade, por exemplo, na Análise do Comportamento, não é exclusivamente 
"individual" e muitomenos "interior",conforme o sentido mentalista (TOURINHO, 1997a;1997b). É assim que a maneira como um indivíduo se vê, como sente, valoriza e descreve a si mesmo, dependendo contexto sócio-cultural caracterizado pelas relações entre os membros de uma comunidade verbal. Mas parece que há, ainda, uma suposta ausência de evidências que justifiquem a presença do hemo socius na Análise do Comportamento. O que se sente é que a especificidade social do comportamento humano continua sendo uma limitação para um intercâmbio teórico-metodológico entre analistas de comportamento e psicólogos sociais na análise do conhecimento social. Porquê? Talvez a falta de visualização das limitações e avanços em cada área - pelos pesquisadores das duas áreas -seja uma das respostas que se procura.

Atualmente, há uma tentativa de recolocar as proposições iniciais de SKINNER, completando-as a partir de dados de estudos mais recentes que, de certa forma, continuam o caminho por ele iniciado. Como não há consenso entre os cientistas sociais acerca do que seja uma prática social (SÁ, 1994), é possível que talvez o nível macro-social esteja sendo considerado pelos analistas de comportamento quando se ocupam das práticas sócio-culturais (LAMAL, 1991). Analistas de comportamento, ao trabalharem com grupos e comunidades, partem do princípio skinneriano (SKINNER, 1983) de que é mais prático mudar a cultura do que o indivíduo, já que as mudanças nas práticas sócio-culturais sobrevivem ao indivíduo. De acordo com PIERCE(1991), os diferentes níveis de análise do comportamento social incluem (1) a análise experimental do comportamento social,

(2) o estudo dos processos e sistemas sociais e

(3) a análise comportamental da cultura e da evolução das práticas culturais (história da cultura). Dessa forma, através das relações conceituais entre contingências, seleção por conseqüências e contexto histórico e atual de reforçamento, os analistas de comportamento têm apontado, a partir de uma análise funcional dos sistemas e práticas sócio-culturais, direções exeqüíveis de alteração nas relações de contingência que mantém as práticas de grupos e comunidades. Em termos mais amplos, isso significa começar "mudando o mundo em que as pessoas vivem" (SKINNER, 1991: 115), já que uma cultura não sobrevive por causa da "mentalidade" dos homens.

Um aspecto interessante que PIERCE (1991) retoma quanto a isso, é o fato de a Análise do Comportamento não dividir a natureza humana em várias. Analistas de comportamento oferecem uma alternativa para a análise das práticas sociais ao postularem que "Princípios do comportamento que explicam o comportamento individual presumivelmente operam quando pessoas agem em um contexto político ou em circunstâncias econômicas" (PIERCE, 1991: 13). É nesse contexto que emerge o conceito de Metacontingência (GLENN, 1991) como referente às relações contingentes entre as práticas sócio-culturais e as conseqüências destas mesmas práticas. Segundo MALOTT (1988), o problema das conseqüências atrasadas típicas das práticas culturais pode ser entendido pelo comportamento governado por regras, já que uma cultura é "prescrita por um conjunto de regras" e que, certamente, caracterizam parte do conhecimento socialmente partilhado por seus membros.

Incursões no sentido de analisar o conhecimento social numa perspectiva comportamental já foram tomadas em tempos anteriores. SÁ (1983) já concluiu, por exemplo, que existem (resguardando as potencialidades diversas) algumas afinidades evidentes entre as proposições de FOUCAULT e SKINNER acerca do saber enquanto comportamentoconhecimento socialmente construído. A fictícia idéia da autonomia humana, por exemplo, é construída em função de uma história de reforçamento individual, por sua vez inserida numa história social; e esta, por sua vez, inserida inerentemente nas práticas de uma comunidade verbal que compõem o locus do reforçamento social dos repertórios descritivos. 
A inserção do comportamento verbal como centro da direção analítica pode ser encontrada em artigos de outros pesquisadores, como maneira de recolocar a relevância potencial da Análise do Comportamento na compreensão dos processos de construção do conhecimento. Uma interessantíssima tentativa nessa direção pode ser vista no artigo de GUERIN (1992a). Segundo este autor, analisar o conhecimento socialmente construído é equivalente a analisar o comportamento das pessoas em grupos, e isso pode ser feito a partir das classes funcionais do comportamento verbal expostas por SKINNER (1978b) e das contingências sociais que as mantêm (as chamadas "práticas sociais" que têm história). Para isso, GUERIN (1992a) retoma autores clássicos da Psicologia Social (MOSCOVICl, JODELET, HERZLICH, MEADe GERGEN) e inicia a sua análise corroborando que o conhecimento social (sobre doenças, teorias psicológicas, ou qualquer outro "objeto social") é aprendido na conversação e interação com outras pessoas, influenciado pela mídia, em subgrupos íntimos ou próximos, ou por conflitos generalizados entre os diversos grupos da amplitude social.

Uma primeira advertência de GUERIN (1992a) é que um conhecimento só é socialmente construído quando é função de contingências ambientais comuns, envolvendo basicamente as formas verbais do tato, do intraverbal e do autoclítico. Com esse aporte, GUERIN (1992a) irá afirmar que o controle do conhecimento socialmente construído se deve às relações sócio-históricas da comunidade verbal e que, atualmente, nas sociedades modernas, a comunidade verbal se encontra difusa e impessoal, por causa do perpasse da mídia. Dessa forma, a "comunidade verbal" é, em menor escala, equivalente aos "coletivos" de DURKHEIM, aos "outros generalizados" de $M E A D$, à "comunidade lingüística" de SAUSSURE e aos "subgrupos" de MOSCOVICl. Assim, o tatear, por exemplo, estaria sob controle dos pequenos grupos que reforçam relatos particulares e punem outros, de modo que as características do grupo constroem (controlam?) o conhecimento do sujeito; e essa construção é mais eficaz quando as conseqüências funcionais forem generalizadas (GUERIN, 1992a).

Uma questão importante é que o comportamento verbal é efetivo pela ação das outras pessoas e usualmente (mas não necessariamente) ocorre na presença de outras pessoas (SKINNER, 1978b; 1982). Isso explicaria a dimensão social da sua construção. Assim, repertórios verbais particulares mantémse em grupos cujos membros agem em contextos discriminativos para a produção desses comportamentos, compartilhando-os no conjunto maior da comunidade verbal que mantém o conjunto da linguagem. Esse seria, segundo GUERIN (1992a), o fundamento behaviorista radical explicativo do comportamento socialmente construído, e se se seguisse o seu argumento, seria possível concluir que na descrição que os psicólogos sociais fazem da construção sócio-histórica de um "modo de conhecer" específico, estaria faltando a análise funcional do controle interpessoal e intergrupal (e por extensão, do controle intrapessoal) que, mediado pela história impregnada na cultura do grupo, explicaria o repertório verbal que caracteriza as temáticas principais nos discursos dos seus membros.

Ao caracterizar a natureza social do conhecimento, é preciso analisar como os elementos de um conhecimento mantém-se no repertório das pessoas em grupos. Assim, é possível que isso ocorra nas seguintes condições (GUERIN, 1992a): (a) se o controle social do tatear os elementos referentes (...) [a um objeto social] for hermeticamente mantido por um grupo - pois ele não fornecerá necessariamente reforçamento de informações corretas; (b) se os intraverbais desse conhecimento dominante for reforçado por um grupo - pois assim eles lembrarão tatos; e (c) se as conseqüências funcionais generalizadas tornarem-se altamente generalizadas - pois também manterão esse tatear generalizado. 
Usando o exemplo do objeto social "AIDS", um portador do vírus HIV pode ter lido, ouvido ou verbalizado que "A AIDS é uma peste gay" e tem sido reforçado por repetir sua afirmação em um contexto verbal particular (o "lido", o "ouvido" e o "verbalizado" são derivados das "condições objetivas"). Sua fala é controlada pelos estímulos contextuais da audiência (lembrando que o falante pode ser o seu próprio ouvinte) e pelas conseqüências que reforçam-na e, embora o seu comportamento seja um intraverbal, ele será reforçado como se se apresentasse como um tato.

É assim que atribuições sobre causas ou origens de doenças (talvez disfarçadas como tatos) podem, num primeiro caso, passar a ser ancoradas na comunidade tornando-se familiares através da conversação; e as informações acuradas sobre as causas de doenças podem, num segundo caso, ser modificadas com as experiências pessoais e diretas com a doença e também com as experiências nas pesquisas médicas (GUERIN, 1992a). No primeiro caso, como regra, informações conversadas podem estar descrevendo contingências fictícias ("Se você fizer sexo de maneira promíscua, então Deus o castigará com a AIDS")que poderão governar o comportamento das pessoas em contato com essas situações. Já no segundo caso, a partir da experiência direta, as contingências modelam o comportamento na sua relação com a saúde (BORLOTI, 1998). Isso insere o conhecimento socialmente construído como comportamento objeto de análise de uma área de pesquisa da ciência do comportamento que examina a diferença entre o comportamento governado por regras e o comportamento controlado por contingências.

Para chegar a isso, é importante analisar - papel das contingências sociais na manutenção do conhecimento social que, na opinião de GUERIN (1992a), tem sido omitido nos estudos da Psicologia Social. Em função disso, GUERIN (1992a) acredita que o papel das conseqüências sociais generalizadas não óbvias, que mantêm a maior parte do comportamento verbal, e o papel do ambiente social em servir duplamente de contexto discriminativo e conseqüente, seriam mais valiosos na explicação do conhecimento socialmente construído, e isso evitaria o raciocínio circular comum na análise das relações entre o cotidiano da comunidade verbal e o conhecimento social, como se estes se explicassem mutuamente.

Para os psicólogos sociais, o conhecimento social emerge como realidade objetiva (SÁ, 1995);e uma realidade é "objetiva" quando ela é uma forte estimulação ambiental que controla o conhecimento acerca dela (GUERIN, 1992a). Isso permite a seguinte conclusão: se os indivíduos vivem em grupos, a análise de seus comportamentos deve considerar aqueles seus determinantes poderosos, seja para a análise de como um repertório verbal forja estímulos discriminativos para outros comportamentos (como no caso das regras verbais que descrevem o comportamento e as contingências que o mantém); seja para a análise do conhecimento social que descreve o comportamento dos indivíduos (WAGNER, 1993), ou seja para a proposição de uma mudança no conhecimento social.

Sempre interessados, em última instância, na modificação do comportamento, os analistas do comportamento também têm se debruçado sobre o papel sutil da mediação conseqüencial entre os indivíduos na modificação do seu conhecimento socialmente partilhado. Esse papel não tem sido mostrado explicitamente nos trabalhos em Psicologia Social (GUERIN, 1992a). Por exemplo, se um analista comportamental retoma as proposições de MOSCOVICI (1981) sobre as minorias, poderá considerar que o poder de influência de uma minoria dependerá das condições oferecidas pelo estabelecimento de uma comunidade verbal consistente (de "minoritários") que reforce um novo conhecimento social, através do uso de argumentos persuasivos, acerca dos efeitos 
reforçadores do novo repertório (o novo conhecimento) ou da indicação da probabilidade de punição na continuação do uso de um conhecimento antigo.

$\mathrm{Na}$ opinião de GUERIN (1992a), argumentos persuasivos e outras formas de organização coletiva compõem o "estilo comportamental" que caracteriza a influência e a transformação social que brota das minorias ativas. Assim, continuando como uso dos termos comportamentais, os comportamentos sociais se transformam a partir de mudanças nas conseqüências generalizadas do tatear para conseqüências específicas que reforçam novos tatos e intraverbais e, posteriormente, autoclíticos. Esse movimentos e efetiva somente porque "A consistência da minoria e outros fatores controladores são métodos de fortalecimento de mudanças nas conseqüências sociais" (GUERIN, 1992: 1430). A crítica de GUERIN (1992b) é que a Psicologia Social se limita a descrever o comportamento verbal compartilhado, sendo que "o papel da comunidade verbal e o envolvimento desta na manutenção destes [comportamentos verbais socialmente construídos] são omitidos na maior parte da análise psicossocial" (p. 600).

Retomando a distinção entre regras e contingências na análise de mudanças sociais, por exemplo, é possível que uma parte da minoria imprima mudanças sociais a partir de comportamentos verbais que especificam as contingências do mundo, sendo que estes servem para trazer à consciência dos ouvintes uma parcela maior da realidade não consciente (SKINNER, 1991) que controla a minoria. Por este meio o grupo pode conseguir que outras pessoas digam coisas que o grupo "socialmente conhece" em interações com outros grupos, inclusive conseguindo e essas outras pessoas refiram-se a si mesmas. Essa "conscientização" atingirá o auge, de acordo com GUERIN (1992a), quando a audiência (essas outras pessoas) fizer contato comas contingências especificadas nos argumentos, de maneira a vira ser reforçada diretamente pelas contingências não verbais.
Um militante sabe disso quando diz que "a 'causa' está no sangue" e por vezes se frustra com a ineficácia do seu trabalho de "conscientização". O que está acontecendo é que o militante pressente que nem toda a minoria terá o seu repertório facilmente governado pelas regras explícitas ou implícitas no seu manifesto.

Analogias consideráveis poderiam ser feitas inserindo esse exemplo nas noções de "cognição quente" e "cognição esfriada" que caracterizariam, respectivamente, entre os psicólogos sociais, as abordagens da Representação Social e da Cognição Social sobre o "modo de conhecer" (MOURA, 1996). Nesse aspecto,tomando outros apontamentos, o que se passa com o militante é que o "sangue" remete às "cognições quentes", visto que são comportamentos controlados por contingências não verbais e as "esfriadas" são assim entendidas por serem comportamentos verbais governados por regras (SKINNER, 1980). Em virtude disso, analistas do comportamento acham um avanço a abordagem do conhecimento social, na vertente da Representação Social, não "construir socialmente" o comportamento verbal como um "depósito de representações mentais", assim como é um avanço situá-lo nas"práticas cotidianas", pois o mesmo não é emitido a partir da sua correspondência direta com o mundo, mas nos seus efeitos primeiros sobre a audiência. Em outras palavras, nas contingências se situa 0 conhecimento social e elas são as suas variáveis controladoras; e elas são geradas por ele como efeitos (SKINNER, 1978b). Contingências impulsionam a emergência do conhecimento social e fazem isso na forma de práticas históricoculturais de uma comunidade verbal, que passam a compor a história individual de reforçamento dos seus membros (SKINNER, 1978b, 1990, 1991; GUERIN, 1992b). Dessa forma, alguns problemas de pesquisa propostos aos analistas de comportamento (salvaguardadas as competências do historiador) são respondidos com a análise histórica sobre os 
valores $\mathrm{e}$ interesses contidos na linguagem como parte de uma cultura (SKINNER, 1983; SKINNER, 1990; TOURINHO, 1993).

Se se pensa assim, os psicólogos sociais, então, ao estudarem um conhecimento social, deveriam estar mais atentos aos modos pelos quais a cultura transmite aos membros esse conhecimento social. Esses "modos" são, na Análise do Comportamento, as contingências sociais no presente dos grupos (SKINNER, 1990; GUERIN, 1992b), especificamente no como os comportamentos de membros de uma comunidade verbal (composta de grupos diversos) seqüenciam mutuamente (e tornamse estímulos discriminativos para os) seus comportamentos na descrição de si mesmos a partir dos outros. Nesse caso, o comportamento verbal torna-se o foco da análise, mas isso aponta em direção a outros problemas neste tipo de repertório.

Talvez o fato de os analistas de comportamento geralmente centrarem suas análises sobre os mantenedores tangíveis dos repertórios verbais que estudam - ao invés das conseqüências generalizadas atravessadas pela mídia - tenha levado os psicólogos sociais (principalmente aqueles influenciados pelo new look da cognição) a acreditarem que conhecimento social, informação, esquema, gramática e outros tantos exemplos de comportamentos verbalmente mediados, não sejam passíveis de uma análise comportamental. Entretanto, é possível afirmar que todos esses comportamentos sob o rótulo de "cognições" normalmente envolvem (GUERIN, 1992b): "(a) comportamentos encobertos, (b) uma história de reforçamento,(c) conseqüências não específicas de uma comunidade verbal $e$ (d) comportamento verbal do tipo autoclítico" (p. 601).

Se conhecimentos sociais surgem das condições objetivas das práticas de um grupo, pode-se concluir que conhecimentos sociais não podem "causar" outros comportamento. Entretanto, um conhecimento social pode ser tomado como parte da manutenção de outros comportamentos, e a explicação (tanto do comportamento mantido, quando do comportamento mantenedor) deve ser buscada nas contingências sociais: últimas instâncias que configuram as práticas sócio-culturais. WAGNER (1993) discute que o conhecimento social tem sido indicado como "explicação" por causa de sua estreita relação com o comportamento social. Esse fato coloca o conhecimento social como uma variável independente, mesmo que sob o rótulo amenizado e levede "guia"do comportamento. $E$ isso tem levantado questões epistemológicas cruciais que valem ser discutidas porque essa mesma questão instiga os analistas de comportamento na análise das regras verbais como "regentes" do comportamento (ZETTLE, 1990).

Em primeiro lugar, WAGNER (1993) lembra que não há consenso entre os psicólogos sociais sobre o papel "causal" do conhecimento construído socialmente. Isso fica evidente em "versões fracas" e "versões fortes" nos estudos das Representações Sociais. WAGNER (1993) parece questionar ambas essas versões ao afirmar que conhecimentos socialmente construídos "(...) não podem servir como explicações do comportamento de indivíduos em sociedade". Com isso, é possível entender que o comportamento descrito no conhecimento socialmente construído é uma "ilustração" do conhecimento (WAGNER, 1993: 237). Um conhecimento social é relevante em uma situação e isso informa sobre o comportamento provável do indivíduo naquela situação; isto pode indicar que o conhecimento governa um outro comportamento relevante. Mas isto não basta para explicar o comportamento relatado (no conhecimento). Talvez seja por essa razão que SÁ (1994) explícita uma preocupação com o nível mais fundamental das relações sociais da vida cotidiana, explorando as riquezas da interpenetração do conceito de práticas sócioculturais com a análise behaviorista radical do comportamento dos indivíduos em grupos. 
SÁ (1994) lembra que as "práticas" "não precisam nem de serem um objeto de reflexão, nem de serem comunicadas de modo explícito para funcionarem efetivamente" (SÃ, 1994:41). É nesse sentido que, talvez, se possa também inserir o conhecimento socialmente construído como tendo gênese nas práticas sociais: apesar de inconscientes, as práticas (ou as contingências sociais?) permitem a explicitação das regras que governam os comportamentos e os julgamentos (valores) implicados nas práticas, por intermédio da linguagem compartilhada. Os conhecimentos sociais dos objetos, nessas práticas, "emergem nesse momento" (SÁ, 1994: 41). E a descrição das relações entre os membros tem uma função na "orientação" dos seus comportamentos.

A justificativa de SÁ (1994) parece atestar a relevância da junção de abordagens teóricas quando ele endossa a entreposição e a comunicação entre os níveis individual e social. É assim que o autor justifica o emprego da Análise Comportamental na discussão que propõe entre as práticas e o comportamento, visto que a perspectiva skinneriana pode ser "particularmente útil" à compreensão dos processos comportamentais básicos nas interações entre o indivíduo e o seu meio social. Além de apontar essa constatação, SÁ (1994) diz que, apesar do conhecimento acadêmico do Behaviorismo geralmente não incluir elementos desse tipo, a rede de contingências de reforçamento social "permite responder" acerca das relações entre as práticas e os fatos sóciohistóricos (p. 43).

$\mathrm{Se}$, de qualquer modo, se quisesse traçar um paralelo entre as práticas sociais e as contingências sociais, seria importante frisar o papel destas em servir de "matéria prima comportamental" ou de "substrato psicológico" para aquelas práticas. Essa matéria prima comportamental, seria responsável pelo controle e determinação do comportamento, não somente em seus aspectos inconscientes, mas também na gênese do conhecimento socialmente construído enquanto práticas verbais. Por isso, SÁ acredita que a distinção entre o Comportamento Modelado por Contingências (como seu caráter inconsciente) e o Comportamento Governado por Regras (emergido do comportamento verbal) propostos por SKINNER (1980) "pode se mostrar especialmente útil à visualização conceitual dessa gênese [social do conhecimento]" (SÁ, 1994: 44).

Esta reiteração de SÁ é mais do que a tentativa de aproximar os conceitos por ele analisados. Apesar da proximidade, Regras não são a mesma coisa que Conhecimentos Sociais, mas "a origem comportamental" destes deve-se às regras verbais que governam 0 comportamento dosindivíduos em práticas sociais específicas (SÁ, 1994).

Comentando o artigo de SÁ (1994), GUERIN (1994) reforça a relevância das argumentações nele contidas, lembrando que 0 Behaviorismo Radical não é uma filosofia sobre o comportamento, mas sim sobre contingências, e essas envolvem o comportamento no contexto da experiência vivida. Ainda, GUERIN chama a atenção para o fato de SÁ descrever de forma precisa o caráter social do Comportamento Governado por Regras, o que habitualmente não tem sido feito pelos analistas de comportamento. De fato, "comportamento governado por regras é um evento social" (GUERIN, 1994: 178) mas só recentemente essa essência tem sido estudada.

GUERIN (1994) acredita que o comportamento tem que ser tomado como explícito e não como conseqüência do pensamento, como apregoa o cognitivismo. Nesse sentido, o olhar dos psicólogos sociais para as práticas sociais é altamente produtivo, mas o termo "práticas"deve ser precisado tendo como foco os comportamentos (não necessariamente conscientes): as comunicações atuais e sistemáticas que ocorrem funcionalmente no cotidiano dos grupos. Para isso, novamente, as categorias de análise do comportamento verbal (SKINNER, 1978b) serão úteis. 
A base do processo de gênese dos conhecimentos socialmente construídos não é imediatamente aparente (GUERIN, 1994). O conhecimento tem, inicialmente, um caráter inconsciente, e tem função na vida cotidiana dos grupos. Isso pode ser encontrado as razões pelas quais alguém conversa com outros ou mantém conversações, já que existem regularidades entre as práticas cotidianas e aquilo que as pessoas dizem para si mesma sou entre si. O comportamento cotidiano envolve eventos inconscientes, pois o sujeito se engaja em práticas sem refletir sobre o que está fazendo. Se essa reflexão ocorre ela se dá, necessariamente, em qualquer caso, com o uso de termos e idéias aprendidas dos grupos sociais. A essa conclusão já se destinou a sugestão de GLENN ao antecipar que as relações entre a análise comportamental e certas abordagens teóricas nas ciências sociais só poderão ser inteiramente discutidas quando uma prática cultural for entendida em termos dos processos comportamentais que a compõe. Nesse entendimento, "A desmistificação do conceito de 'regras' pode providenciar a construção de uma ponte que é necessária" (GLENN, 1987: 32).

$\mathrm{O}$ que se conclui dos argumentos dos psicólogos sociais é que o psicossocial é conceituado como o fenômeno emergido e materializado a partir das relações entre os sujeitos e a sua realidade social, e explicita, por excelência, as relações entre o social e o subjetivo (DEL PRETTE, 1994). Poderia ser dito, igualmente, que o comportamental social é conceituado como fenômeno adquirido, mantido e modificado a partir das relações dos sujeitos com sua realidade social e também explicita as relações entre o social e o subjetivo, já que o privado está relacionado às práticas características de uma cultura (TOURINHO, 1997b). Com isso, analistas de comportamento admitem "a própria constituição social da subjetividade de cada um, na medida em que cada um vem a experimentar certos sentimentos enquanto tais, a partir da 'interação com um dado grupo social (TOURINHO, 1997a: 181).

\section{REFERÊNCIAS BIBLIOGRAFIA}

BORLOTI, E. B. (1998). Regras e Identidade na Condição de "Portador do HIV/AIDS": Integrando a Análise do Comportamento e a Psicologia Social. Dissertação de Mestrado, Universidade Federal do Espírito Santo.

DEL PRETTE, A. (1994). A Psicologia Social e a Análise do Fato Social. Psicologia: Reflexão e Crítica. Porto Alegre, v. 7, n. 2, 125:132.

GLENN, S. S. (1987). Rules as Environmental Events. The Analysis of Verbal Behavior, 5, 29:32.

GLENN, S. S. (1991). Contingencies and Metacontingencies: Relations Among Behavioral, Cultural, and Biological Evolution. In.: LAMAL, P. A. (Ed.). (1991). Behavioral Analysis 01 Societies and Cultural Practices. Washington: Hemisphere Publishing Corp., 39:73.

GUERIN, B. (1992a). Behavior Analysis and the Social Construction of Knowledge. American Psychologist, v. 47, n. 11, 1423:1432.

GUERIN, B. (1992b). BehaviorAnalysis and Social Psychology: A Review of Lana's Assumptions of Social Psychology. Journal of the Experimental Analysis of Behavior. v. 58, n. 3, nov., 589:604.

GUERIN, B. (1994). Using Social Representations to Negotiate the Social Practices of Life: Commentary on the Paper by C. P. de Sá. Papers on Social Representations, v. 3, n. 2, 177:183.

LAMAL, P. A. (Ed.). (1992). Behavioral Analysis 01 Societies and Cultural Practices. Washington: Hemisphere Publishing Corpo

MALOTT, R. W. (1988). Rule-Governed Behavior and Behavioral Antropology. The Behavioral Analyst, v. 11, n. 2, 181:203. 
MICHELETTO,N. \& SÉRIO, T.M.A.P. (1993). Homem:Objetoou Sujeito paraSkinner? Temas em Psicologia, São Paulo, 2, 11:21.

MOSCOVICI,S. (1978). A Representação Social da Psicanálise. Rio de Janeiro: Zahar.

MOSCOVICI,S. (1981). Pslcología de las Minorias Actlvas. Madrid: Ediciones Morata.

MOURA, M. L. S. (1996). Práticas SócioCulturais, Construção Compartilhada de Conhecimento e Representações Sociais. In Z.A. TRINDADE \& C. CAMINO (Orgs.) Cognição Social e Juízo Moral. Coletâneas da ANPEPP, v. 1, n. 6, 25:44.

OVERSKEID, G. (1995). Cognitivist or Behaviourist - Who Can Tell the Difference? The Case of Implicit and Explicit Knowledge. Britlsh Jounal of Psychology, 86, 517:522.

PIERCE,W. D. (1991).Cultureand Society: The Role of BehavioralAnalysis.In.: P.A. LAMAL (Ed.) (1991). Behavioral Analysis of Societies and Cultural Practices. Washington: Hemisphere Publishing Corp. 13:37.

SÁ, C. P. de. (1983). Sobre o Poder em Foucault e o Controle em Skinner. Arquivos Brasileiros de Psicologia. Rio de Janeiro, v. 35, n. 2, 136:145.

SÁ, C. P. de. (1994). Sur Les Relations Entre Représentations Sociales, Pratiques Socio-Culturelles Et Comportement. Papers on Social Representations, v.3. N. 1, 40:46.

SÁ, C.P. de. (1995). Representações Sociais: o Conceito e o Estado Atual da Teoria. In M. J. SPINK (Org.). O Conhecimento no Cotidiano: as Representações Sociais na Perspectiva da Psicologia Social. São Paulo: Brasiliense, 19:45.
SKINNER, B.F.(1978a). Ciência e Comportamento Humano. São Paulo: Martins Fontes.

SKINNER, B.F. (1978b). O Comportamento Verbal.São Paulo:Cultrix: Edusp.

SKINNER, B.F. (1980). Contingências do Reforço: Uma Análise Teórica. São Paulo: Abril Cultural (Coleção "Os Pensadores").

SKINNER, B. F. (1983). O Mito da Liberdade. São Paulo: Summus.

SKINNER, B. F. (1990). Can Psychology be a Science of Mind? American Psychologist, V. 45, n. 11, 1206:1210.

SKINNER,B.F. (1991). Questões Recentes na Análise Comportamental. Campinas, S.P.: Papirus.

TOURINHO,E. Z. (1993). O Autoconhecimento na Psicologia Comportamental de B. F. Skinner. Belém, PA: UFPA, $\mathrm{CFCH}$.

TOURINHO, E.Z. (1997a). Eventos Privados em uma Ciência do Comportamento. In. BANACO, R. A. (Org.). (1997a). Sobre Cognição e Comportamento: Aspectos Teóricos, Metodológicos e de Formação em Análise do Comportamento e Terapia Cognitivista.Santo André, SP.: ARBytes.

TOURINHO,E. Z. (1997b). O Conceito de Comportamento Encoberto no Behaviorismo Radicalde B. F. Skinner. In. R.A. BANACO, (Org.). (1997b). Sobre Cognição e Comportamento: Aspectos Teóricos, Metodológicos e de Formação em Análise do Comportamentoe Terapia Cognitivista. Santo André, SP.: ARBytes.

TOURINHO, E. Z. (1997c). Privacidade, Comportamento e o Conceito de Ambiente Interno. In. R.A. BANACO(Org.). (1997c). Sobre Cognlção e Comportamento: Aspectos Teóricos, Metodológicos e de 
Formação em Análise do Comportamento e Terapia Cognitivista. Santo André, SP.: ARBytes.

WAGNER, W. (1993). Can Representations Explain Social Behaviour? A Discussion of Social Representations as Rational
Systems. Papers on Social Representations, v. 2, n. 3, 236:249.

ZETTLE, R. D. (1990). Rule-Governed Behavior: A Radical Behavioral Answer to the Cognitive Challenge. The Psychological Record, v. 40, n. 1,41:49. 